\title{
CHAOS THEORY: IMPACT ON AND APPLICATIONS IN MEDICINE
}

\author{
Arunachalam Kumar \\ Professor of Anatomy, K.S. Hegde M edical Academy, Nitte University \\ M angalore - 575 018, India \\ Correspondence: \\ Arunachalam Kumar \\ E-mail : editornujhs@nitte edu.in
}

\begin{abstract}
:
The advent of a revolutionary and exciting theory that proposes non-linear models as effective adjuncts to linear mathematics in interpreting long-held scientific tenets has provided novel and innovative designs and methodologies that help the medical world to better understand inferences from laboratory investigations, physiological processes, pharmarmaco-therapeutics and clinical diagnostics.

This overview outlines a few salient areas in medicine that have successfully applied the principles of the chaos theory. Chaotic systems have been shown to operate in quite a few physiological processes. The impact and implications of the new science on the future course of medical diagnostics and health science systems as a whole cannot be overstressed.
\end{abstract}

Keywords: chaostheory, non-linear mathematics, fractal, randomness

\section{Introduction:}

Lorenz an American mathematician and meteorologist was the pioneer of the chaos theory. He studied weather patterns he began to realize that they did not always change as predicted. M inute variations in the initial values of variables in his twelve-variable computer weather model would result in grossly divergent weather patterns. This sensitive dependence on initial conditions came to be known as the butterfly effect ${ }^{1,2}$

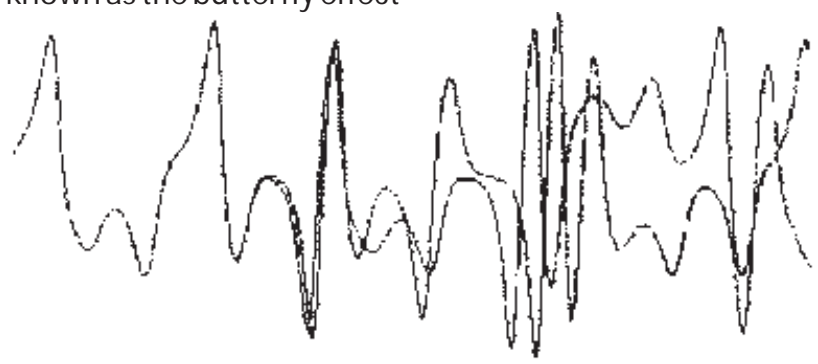

Figure 1: Lorenz's experiment: the difference between the starting values of these curves is only .000127 . (Ian Stewart, Does God Play Dice? The Mathematics of Chaos, pg. 141)

\begin{tabular}{|c|}
\hline Access this article online \\
\hline Quick Response Code \\
\hline \\
\end{tabular}

and pharmaco-therapeutic process involved in health science is dictated by direct Newtonian tenets of cause effect and linear mathematical derivations. Exclusive and total dependence on scalar models have compartmentalized patient profiles into rigid cocoons such as age, sex, weight, height, physiological and biochemical evaluations making interventional therapeutic regimens solely dictated by our over-riding choice to use scales of convenience for every single one of our evaluation, assessment and treatment criteria.

The new field of theoretical biology, spawned by the advent and ready acceptance of the 'chaos' model has lead to an explosion in nascent schools of thought that challenge hitherto entrenched linear mathematical Newtonian and Euclidian principles in natural sciences. Fractal configurations and such other non-linear systems have made the need for a second look at physio-dynamic processes of the human biology, mandatory. Despite rapid strides in the alternate chaotic and randomness applications, the world of medicine is still loathe to modify its dependence and confidence in pharmacokinetic therapeutic solutions based on cause-effect and Euclidean extrapolation linear systems, both in diagnosis and treatment. 
The theory, an emerging branch of mathematics, has since found application in diverse scientific streams. In essence, the hypothesis postulates that even minuscule and minor variations or differences in initial inputs, over a period of time, can and do lead to extraordinary differences in final output. In effect, wherever and whenever one uses rounded-off or approximated values for calculating outcomes, one errs. The implication of this tenet is monumental in the world of medicine - where, almost all inferences, pharmaco-therapeutic regimens and clinical interventions are essentially and exclusively based on averages, means and approximations. Drugs, dosages or surgical procedures are based on diagnosis, which itself is deduced from age, weight, height, readings and almost all values in clinical and laboratory investigations: the value/s of each one of the listed factors being expressed as 'ranges', averages or approximations.

\section{Chaos: Cardiology}

The human heart also has a chaotic pattern. The time between beats does not remain constant; it depends on how much activity a person is doing, among other things. Under certain conditions, the heartbeat can speed up. Under different conditions, the heart beats erratically. It might even be called a chaotic heartbeat. The analysis of a heartbeat can help medical researchers find ways to put an abnormal heartbeat back into a steady state, instead of uncontrolled chaos ${ }^{3}$

Using normal ECG recordings and measuring the just the QR spike height wide fluctuations it was observed at 100 times scale, demonstrates the absolute randomness in the electrical activity of the heart even within a single cardiac cycle. The erratic heights, representing the milli-volt electrical impulse from the sinu-atrial node clearly demonstrate that randomness or chaotic behavior is norm and signifies good cardiac health. Indeed, by analogy, the ECGs should be 'most' regular in only when static, as in death - a fact well demonstrated in the absolute flat ECG record of the dead human heart. The paradoxical and skewed results in seemingly normal processes, when viewed through 'chaos' systems, show how wrong our total reliance on scale and linear applications are! Simplistic interpretations such as this throw up more dilemmas.

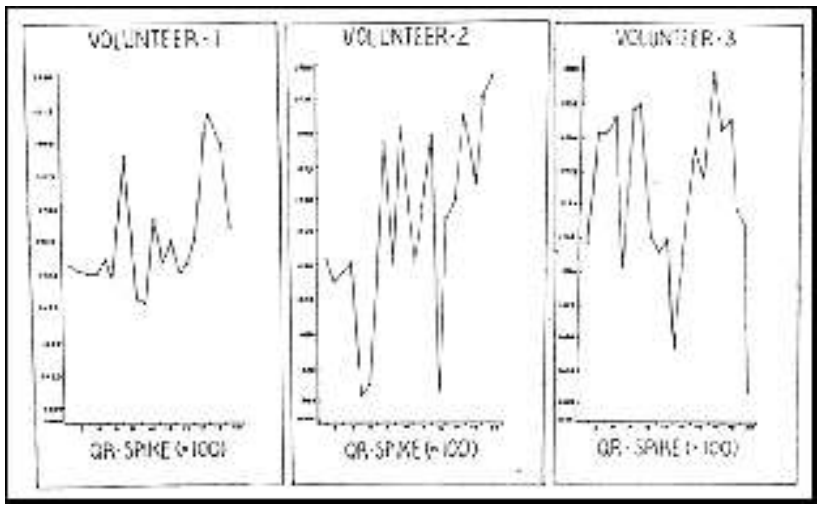

Fig. 2: Q-R spike heights in normal ECGs (x 100) showing chaotic fluctuations and randomness

Research has demonstrated that a reduced standard deviation of heartbeat intervals can predict increased mortality in a group of cardiac subjects, each of which has a reduced standard deviation, but it cannot specify which individuals will or will not manifest lethal arrhythmogenesis $^{4}$

In a report, observations identical to that from an earlier work of Kumar ${ }^{5}$, Poon \& Merill ${ }^{6}$ (1997) analyzed of electrocardiograms from healthy subjects and those with severe congestive heart failure show short-term variations of beat-to-beat interval exhibited strongly and consistently chaotic behavior in all healthy subjects, but were frequently interrupted by periods of seemingly nonchaotic fluctuations in patients with congestive heart failure. Chaotic dynamics in the data collated, exhibited a high degree of random variability over time, suggesting a weaker form of chaos. These findings suggest that cardiac chaos is prevalent in healthy heart, and a decrease in such chaos may be indicative of Congestive Heart failure.

However, more sensitive instruments reveal that normal heart rhythm shows small variability in the interval between beats, ${ }^{7,}$. Our hearts rarely beat the same way twice. Varying opinions on role of randomness and chaos have been proposed, among which one standing ground is that heart function is non chaotic when healthy and turns erratic, with creation of spatial chaos. Kumar ${ }^{9}$ however reports that even in normal state, heart physiology and 
function is actually chaotic, and when these attributes become less random or chaotic, cardiac dysfunction manifests.

\section{Chaos: Thermoregulation in the testes}

The free suspension of the testicles in a loosely wrapped and hyper mobile sac, contributes to a random swing of the organs. The involuntary up, down, anterior, posterior, right or left, circular and elliptical motions that the hung scrotum exhibits, continuously, induces a chaotic cooling system to operate. The non-volitional erratic excursions of the freely suspended sac, allows for better exposure and fanning of its contents to ambient environmental temperature, more effectively. The scrotum is a classic example of natural chaotic cooling system, and that this may well be the primary contributor to thermoregulatory mechanisms in the metabolically active gonad ${ }^{10}$

That the testicles are pulled up, involuntarily, if and when temperatures go below optimal levels, through a reflex muscular contraction, the retracted scrotum becomes less mobile and more 46

fixed or, far less chaotic in movement. This control of erratic excursions and the subsequent inhibition of the chaotic cooling system, arrests continued cooling. The question is, could prolonged and continuous restrictive apparel lead to more serious complications than just azoospermia or oligospermia? Could limiting the chaotic cooling trigger malignant testicular tumors?

\section{Chaos: breast cancers}

The mammary gland in the mammalian female is also subject to similar 'chaotic cooling system for temperature regulation. Apart from the testicles, the only other involuntarily freely mobile organ in the human is the mammary gland in the female. The breasts too, much like the scrotal sac, is by nature erratically and randomly mobile: Attempts to restrict its mobility through the common and widely prevalent use of brassieres, compromises its chaotic cooling ability, consequence of which is derangement of its temperature regulatory mechanisms. Even the development of breast cancers have been attributed to the compromise or restriction of the normal random and erratic non-voluntary movements of these organs ${ }^{10}$

\section{Chaos: Genetics}

In health there is diversity and variety, and in disease that range is narrowed. The more pronounced or long standing an affliction is, the less variation in facial features. Astoundingly too, disturbed hormonal levels too appear to erode physical features, adrenocorticoid disturbances produce Cushing's and every medical of second year can correctly ID a Cushing

syndrome without batting an eyelid. Pituitary gland problems, dwarfism, gigantism, achondroplasia. . .the tell tale face: why, even prolonged exposure to bacteria, toxins or poisonous substances too to engender such a an effect : leonine face of Hansens, lantern jaw in those handling phosphorous, depressed nasal bridge of congenital syphilis ${ }^{11}$.

In fact, many here will be able to recall one condition or other where they have found facial similarities. Craniometry, accurate measurements of the skull appears to hold the key to disease and its diagnosis. This facefeature variation/uniformity hypothesis of mine needs much more documentation and effort. I am fascinated by its effect and implication. With a little refinement and proper record maintenance, one could spot a patient with syphilis, cancer or HIV just by observing merely by checklisting features that are common. To me the entire facial morphometrics, its variation in normal health and erosion in disease mirrors the postulations of the 'Chaos Theory'

Extrapolating, one can even interpret the mutations that engender evolution. Variety is perpetuation of the species: stagnation, as is evidenced in the 'face uniformity' in evidently confers a recognizable template visage and physique (phenotype). Genetic pool permutations are not infinite as the template gene is finite and numbered. When the stage of gene pool saturation is neared or reached, mutations occur: with spontaneous mutations, the total available gene pool is once again multiplied many times 
over, allowing for new combinations of genes - allowing evolutionary biology to spawn new species and subspecies $^{12}$.

The theory proposed here is that infancy, senility, or disease (congenital or acquired and chronic) reduce resistance, lower immunity and compromise health systems ${ }^{13}$. Further, the functioning of cerebration is compromised hugely in any one of the above listed conditions. Memory loss, inability to control sphincters, susceptibility to infections, demonstrate that 'chaotic' randomness is health and randomness or erraticity are evolutionary norms. Reduce the variability quantum increase the chances of disease.

\section{Chaos: Neurology \& gerontology}

Specialists in non-linear dynamics are doing their best to understand the workings of the brain, heart and immune system using chaos theory. Chaos in the brain is a sign of health, not disease. During an epileptic seizure, the disorderly jumble of brain activity suddenly becomes abnormally regular. Preliminary experiments that suggest that the erratic activity of white blood cells is a form of chaotic behavior ${ }^{14}$

By applying chaos theory's mathematical predictions to events such as proteolysis or lipid peroxidation and by using concepts based on fractals to explore the structural changes which happen with ageing, a better insight can be gained into the details of these mechanisms ${ }^{15}$

$M$ any age-related processes can now be quantified and the effects of the treatment can easily be anticipated. For example, in stroke with arm paralysis, it would be functionally advantageous to re-establish an increase of the range of movement of the arm joints (i.e., to increase the fractal, space-filling dimension of the movement), rather than merely increase the strength of the muscles. Conventional treatment with extraneous hormones aims at re-establishing the deficiency of the hormone (a linear approach), but contributes little to the synchronicity of the secretion. If the frequency of the stimulus is incorrect, then a response will not take place, or it will occur sub-optimally.
Therefore, it is necessary to use non-linear approaches during hormonal replacement therapies. Simple linear processes can also be influenced but the result may not always be remarkable or clinically relevant ${ }^{16}$

\section{Chaos: Anatomy and physical anthropology}

Chaos is evident in structural and functional human anatomy. The human foot is admirably adapted to withstand the heavy demands that bipedal gait imposes on it. This compact mass of bones, muscles, fascia and ligaments is superbly adapted to biped locomotion and stance. In this brief study we report out observations on the changes, if any, in weight bearing adaptations and modulations of feet caused uninterrupted and prolonged standing. We continuously monitored footprint pattern alterations and changes in an adult male volunteer. Our inferences show that, that the weight bearing surface of the foot, the sole, constantly varies its area of surface contact during extended weight bearing.

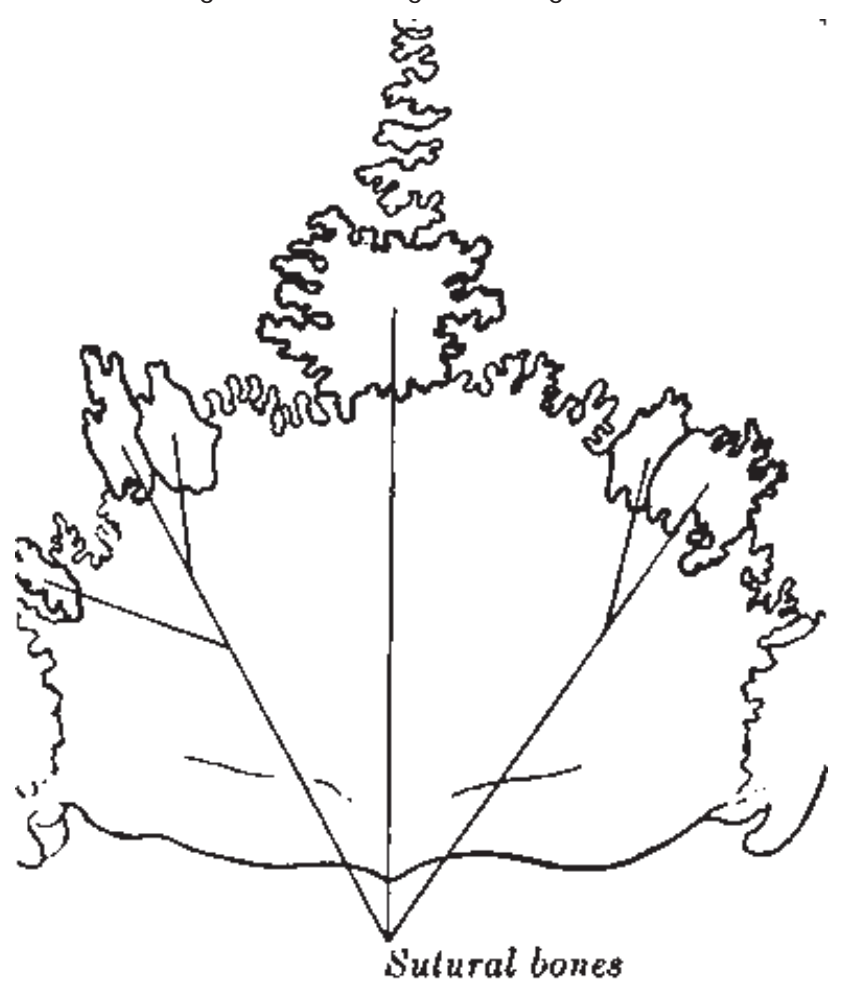

Fig. 3: Cranial sutures

(From: http://www.prohealthsys.com/anatomy/grays/osteology/sutural_bones.php)

The constant shifting and altering in different points of weight transfers are best explained through application of the principles of chaos theory. The random and irregular 
variations show that the science of chaos plays a vital role in extended bipedal static stance and maintenance of posture by randomly and continuously re-distributing foci of weight-bearing on the soles of feet ${ }^{17}$

Anatomically, the strongest joints are the sutural - where the number of protrusions and indentations on the edges of the abutting bones dovetail dramatically to raise the total length of contact between bone and bone. This understanding could have a huge impact on fracture healing, unions or non-unions

Fractal formulations are found more often in human anatomy than we acknowledge, whether it be the branching pattern of an artery or the division of the bronchial tree, extensions of chaotic science through fractal science, topology or cusp-catastrophic models are rife in human structure and function ${ }^{17,18}$.

\section{Chaos: Endocrinology}

As an extension of the above study, application of chaos in the development of diabetic neuropathies and foot ulcers have been identified months earlier with a remarkable degree of accuracy by some researchers analyzing weightbearing capabilities of the foot. Innovative appliances using computer generated graphical data on serial comparisons of 'time series analysis' of microcirculation (or its compromise) on a multiplicity of weight bearing foci on the sole of the foot applying mathematical models ${ }^{19,20}$.
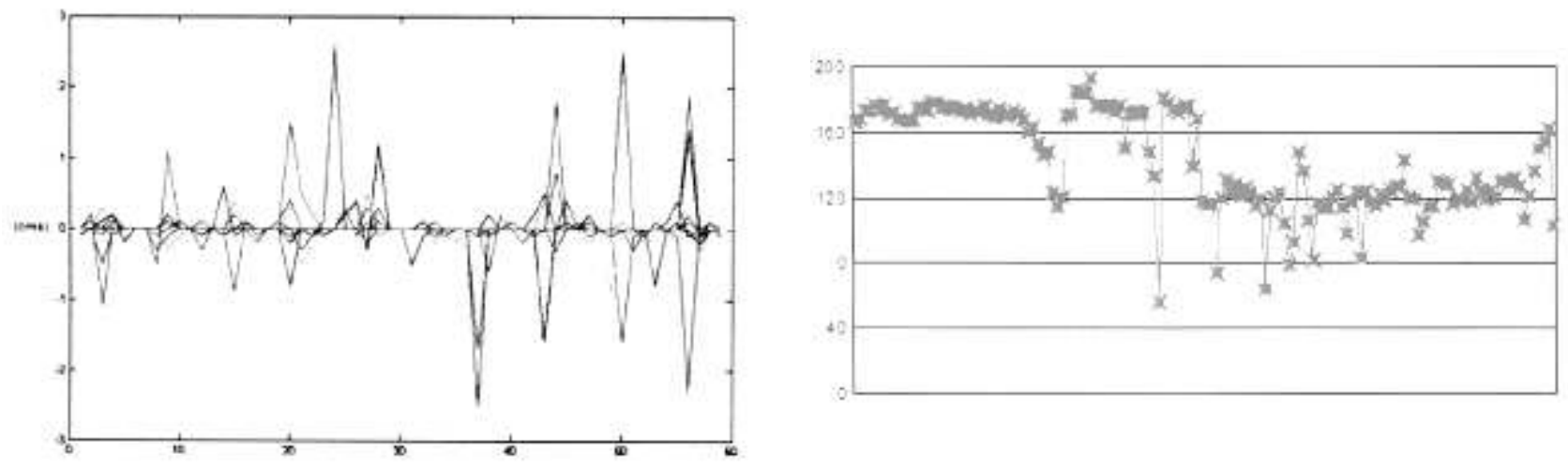

Fig. $5 \& 6$ : Time series graphs and table of microcirculation on foot sole in healthy individuals. Note chaotic pattern of changes in capillary vascular flow levels (courtesy: Kumar C) \& www.Avis-healthcare.com)
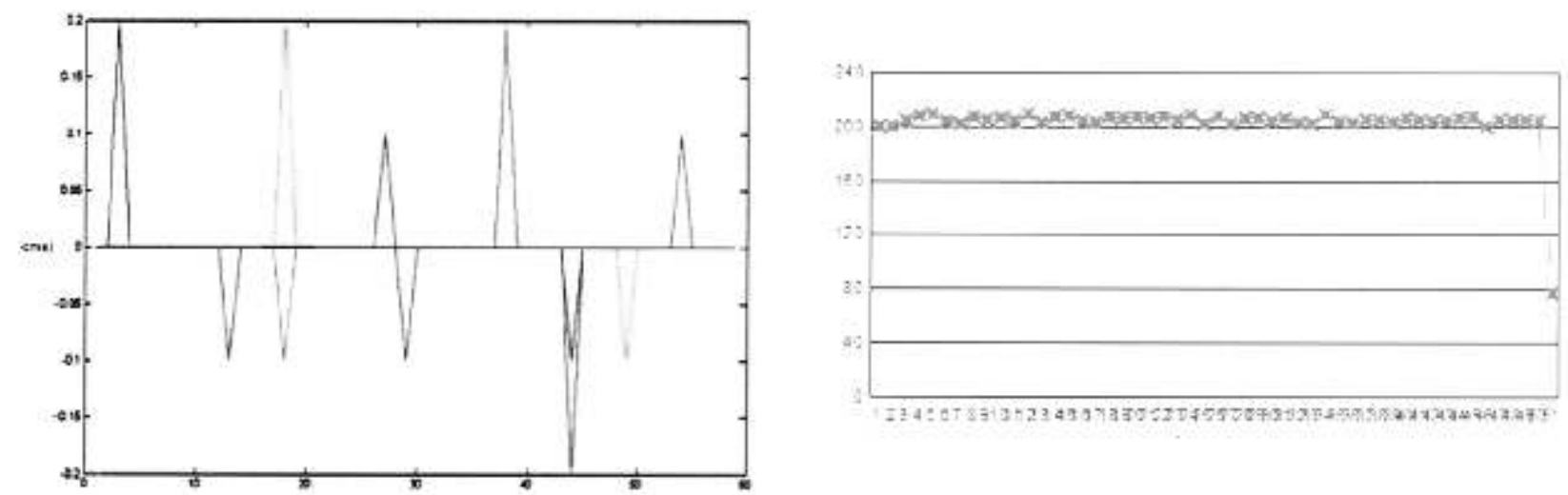

Fig. 6 \& 7: Graphs on microcirculation on foot sole in diabetic individual showing reduction in randomness and range of fluctuation of capillary vascular flow. (courtesy: Kumar C] \& www.AvIS-healthcare.com) 


\begin{tabular}{|c|c|c|c|c|c|c|c|c|c|c|c|c|}
\hline \multirow[t]{2}{*}{ Min } & \multirow{2}{*}{$\begin{array}{l}\text { Meyer's } \\
\text { line }\end{array}$} & \multirow{2}{*}{$\begin{array}{l}\text { 2nd } \\
\text { toe }\end{array}$} & \multirow{2}{*}{$\begin{array}{l}\text { 3rd } \\
\text { toe }\end{array}$} & \multirow{2}{*}{$\begin{array}{l}\text { 4th } \\
\text { toe }\end{array}$} & \multirow{2}{*}{$\begin{array}{l}5 \text { th } \\
\text { toe }\end{array}$} & \multicolumn{2}{|c|}{$2 \mathrm{~cm}$} & \multirow{2}{*}{\begin{tabular}{|c|} 
Mid point \\
left
\end{tabular}} & \multicolumn{2}{|c|}{ Mid point } & \multicolumn{2}{|c|}{ Limits of pale area } \\
\hline & & & & & & left & right & & left & right & lower & upper \\
\hline 1 & 12.9 & 1.6 & 2 & 2.4 & 2.7 & 0.4 & 0.8 & 0.7 & 0.6 & 0.3 & 0.8 & 10.3 \\
\hline 2 & 12.8 & 1.5 & 2 & 2.3 & 2.8 & 0.4 & 0.8 & 0.5 & 0.3 & 0.1 & 1.3 & 10.3 \\
\hline 3 & 12.9 & 1.4 & 2.1 & 2.4 & 2.3 & 0.5 & 1.3 & 0.7 & 0 & 0 & 0.6 & 9.8 \\
\hline 4 & 12.8 & 1.5 & 2.2 & 2.5 & 3 & 0.3 & 0.4 & 0.7 & 0.4 & 0.4 & 1.2 & 10.3 \\
\hline 5 & 12.8 & 0 & 0 & 2.5 & 2.4 & 0.4 & 0.8 & 0.7 & 0.1 & 0 & 1 & 10.4 \\
\hline 6 & 12.8 & 0 & 0 & 0 & 0 & 0.7 & 1.3 & 0 & 0 & 0 & 0.4 & 8 \\
\hline 7 & 12.9 & 0 & 0 & 2.5 & 3 & 0.7 & 0.8 & 0.8 & 0 & 0 & 0.7 & 10.2 \\
\hline 8 & 12.8 & 1.5 & 2 & 2.4 & 2.4 & 0.5 & 0.7 & 0.8 & 0 & 0 & 1 & 10.2 \\
\hline 9 & 12.9 & 1.4 & 2.1 & 2.4 & 2.7 & 0.5 & 1.1 & 0.8 & 1.4 & 0 & 0.2 & 7.5 \\
\hline 10 & 12.8 & 0 & 0 & 2.3 & 2.7 & 0.8 & 0.9 & 0.8 & 0.5 & 0 & 0.5 & 10 \\
\hline
\end{tabular}

\begin{tabular}{|c|c|c|c|c|c|c|c|c|c|c|}
\hline \multirow[t]{2}{*}{ Min } & \multirow{2}{*}{$\begin{array}{l}\text { Meyer's } \\
\text { line }\end{array}$} & \multirow{2}{*}{$\begin{array}{l}\text { 2nd } \\
\text { toe }\end{array}$} & \multirow{2}{*}{$\begin{array}{l}\text { 3rd } \\
\text { toe }\end{array}$} & \multirow{2}{*}{$\begin{array}{l}\text { 5th } \\
\text { toe }\end{array}$} & \multicolumn{2}{|c|}{$2 \mathrm{~cm}$} & \multicolumn{2}{|c|}{ Great toe } & \multicolumn{2}{|c|}{ Limits of pale area } \\
\hline & & & & & left & right & left & right & lower & upper \\
\hline 1 & 14 & 1.8 & 2.6 & 3.3 & 0.9 & 1.8 & 0.6 & 0.6 & 0 & 11.2 \\
\hline 2 & 14 & 1.8 & 2.6 & 3.3 & 1.0 & 1.7 & 0.6 & 0.6 & 0 & 11.2 \\
\hline 3 & 14.1 & 1.6 & 2.5 & 3.2 & 1 & 1.7 & 0.6 & 0.7 & 0 & 11.2 \\
\hline 4 & 14.1 & 1.6 & 2.6 & 3.4 & 1.1 & 1.7 & 0.5 & 0.6 & 0 & 11.2 \\
\hline 5 & 14.2 & 1.6 & 2.4 & 3.2 & 1 & 1.7 & 0.6 & 0.6 & 0 & 11.2 \\
\hline 6 & 14 & 1.5 & 2.3 & 3.1 & 1.1 & 1.6 & 0.6 & 0.6 & 0 & 11.2 \\
\hline 7 & 14 & 1.5 & 2.3 & 3.3 & 1.2 & 1.5 & 0.6 & 0.6 & 0 & 11.2 \\
\hline 8 & 14 & 1.6 & 2.4 & 3.2 & 1.2 & 1.5 & 0.6 & 0.7 & 0 & 11.2 \\
\hline 9 & 14 & 1.6 & 2.4 & 3.4 & 1.2 & 1.5 & 0.6 & 0.6 & 0 & 11.2 \\
\hline 10 & 14 & 1.6 & 2.4 & 3.6 & 1.3 & 1.5 & 0.6 & 0.6 & 0 & 11.2 \\
\hline
\end{tabular}

Table $1 \&$ 2: Show narrowed range of fluctuations in values collated in health individuals and wide range of fluctuations (chaotic) readings in diabetics

\section{Chaos: Surgery}

In surgery, N-plasty, W-plasty and M-plasty techniques have been in vogue for long. Irregular lengthening of an incision or scar has been seen to produce better results, both surgically and aesthetically. Why should a laceration heal better when its edges jagged or serrate and not aligned straight and side by side has been a question never raised or answered by pathologists or surgeons. Unknowing plastic surgery had been applying techniques described as chaos systems.

\section{Scar}

Incisions for W-Plasty (around the scar)

\section{Closure of W-Plasty}

Fig: 7: Modifications in suturing techniques as extended applications of 'chaos'

(From: http://lamfacialplastics.com/wp-content/uploads/2009/05/w-plasty.gif)
The reason why N, W or M procedures produce less scar tissue could be due to operation of the chaos theory - the more the irregular the input the healthier the output.

\section{Chaos: Physiology}

According to some, even cyclical and periodic physiological processes such as menstruation including the transition to menopause, results from a specific kind of complex system, namely, one that is nonlinear, dynamical, and chaotic ${ }^{21}$.

The dynamics of fluid flow and turbulence are areas that have engaged medical researchers applying 'chaotic systems' to study cardiovascular physiology and the biophysics of blood flow. In fact, this area has much potential for research in the world of medicine. Blood pressure and the heamo-dynamics of vascular biomechanisms throw up challenges that defy conventional physics and linear mathematical analyses. Cerebral and renal circulatory physiology and their alterations remain 
yet to be fully unraveled or understood. Chaos may prove to be the answer to many vexing questions.

\section{Chaos: Dermatology}

In dermatology, an initial assessment of lesion symmetry is performed. Only asymmetrical lesions are then fully assessed. This triage works because the hallmark of malignancy is disordered growth that nearly always equates to a lesion growing asymmetrically ${ }^{22}$.

\section{Conclusions:}

Although chaos is often thought to refer to randomness and lack of order, it is more accurate to think of it as an erratic randomness that results from complex systems and interactions among systems. (The concept is revolutionary

\section{References:}

1. Edward N. Lorenz (1969). "Atmospheric predictability as revealed by naturally occurring analogues". Journal of the Atmospheric Sciences $26(4): 636-646$.

2. Tim Palmer (2008); 'Edward Norton Lorenz' Physics Today 61 (9): 81-82 M arkham F W (1998); A method for introducing the concepts of chaos theory to medical Students; Theor M ed Bioeth., 19(1):1-4.

3. Chaos theory: a brief introduction, http://www.imho.com/grae /chaos/chaos.html

4. Skinner J. E., M olnar M, Vybiral T and Mitra M (1992), Application of chaos theory to biology and medicine Integrative Physiological and Behavioral Science, Volume 27, Number 1, 39-53, DOI: 10.1007/ BF02691091

5. Kumar J C \& Arunachalam Kumar (2002), Chaos science \& weight bearing foci in the biped, National Conference of the Anatomical Society of India http:// medind.nic.in/jae/t05/i1/jaet05i1p32.pdf

6. Chi-Sang Poon \& Christopher K. Merrill (1997), Decrease of cardiac chaos in congestive heart failure Nature 389, 492-495: doi:10.1038/39043

7. Arunachalam Kumar, Hegde B. M. \& Prabhu. S.P. (1996); The chaos theory, cardiology \& electrocardiographs; Proceedings of the National Conference on Chaos Theory, Bangalore

8. Rao A, Kumar Arunachalam Kumar (1998) Intrinsic cardiac randomness and diagnostic pitfalls in electrocardiography. In: Niranjan UC, editors. Proceedings of the National Conference on Biomedical Engineering

9. Arunachalam Kumar (2011), Chaos \& electrocardiography, Nitte University Journal of Health Science, Vol.1, No.4

10. Arunachalam Kumar (2009), Burn the bra: compromised chaotic cooling as cause for testicular and breast carcinomas, Medical Hypotheses, 73 (6) 1079-80, 2009 in that it requires us to adopt a different frame of reference which, at times, may move us away from previous concerns and methods of data analysis.

Chaos has already had a lasting effect on science, yet there is much still left to be discovered. Many scientists believe that twentieth century science will be known for only three theories: relativity, quantum mechanics, and chaos. Aspects of chaos show up in the fractal blood vessels to the branches of trees and the effects of turbulence: However, a word of caution is mandated here: Although it is easy to get swept and go overboard over the impact and applications of 'Chaos Theory' - for the theory it still remains just that, a theory.

11. Arunachalam Kumar \& Kumar JC (2010), The face as an index in health and disease, M ed. Hypotheses, 74(2):389-90

12. Arunachalam Kumar, 'Kumar's gene saturation theory' Parts I \& I| http://network.nature.com/groups/transcience/forum/topics/5277/ http://network.nature.com/groups/transcience/forum/topics/5365

13. Pyne John, Alan Cameron A, Rosendahl C, http://www. medicalobserver. com.au/news/chaos-theory-in-practice

14. Marios Kyriazis (2003), Practical applications of chaos theory to the modulation of human ageing: nature prefers chaos to regularity, Biogerontology 4: 75-90

15. The Economist, September 18th, 1999. http:// www.economist.com

16. Pritchard WS (1992); The brain in fractal time. Intern J Neurosc 59:945-949

17. Briggs, John. Fractals: the Patterns of Chaos. Touchstone, Simon and Schuster Inc. New York, NY. 1992.

18. Ives C, Human beings as Chaotic Systems, http:// www.fractal.org/LifeScience-Technology/Publications/Human-beings-as-fractalsystems.pdf

19. Oberoi DV, Kumar JC, Arunachalam Kumar, D'Souza S \& Hegde B. M. (2007). Does non-chaotic weight - bearing foci cause foot ulcerations in diabetics? M ed Hypotheses, 68(2):468 - 9.

20. Kumar JC, Srikumar K; A medically researched solution to avert foot ulcers \& diabetic neuropathy complications: Nebula CJ 95: AVIShealthcare

21.Paula S. Derry, Gregory N. Derry (2012), Menstruation, Perimenopause, and Chaos Theory, Perspectives in Biology and Medicine, Volume 55, Number 1, pp. 26-42 | 10.1353/pbm.2012.0003

22. Bulinska A, http://www.medicalobserver.com.au/news/pathologyof-pigmented-lesions 